\title{
Is there association between Glutathione $S$ Transferases polymorphisms and cataract risk: a meta-analysis?
}

Wen Sun ${ }^{1 \dagger}$, Liling Su${ }^{2+}$, Yan Sheng ${ }^{1}$, Ye Shen ${ }^{1 *}$ and Guangdi Chen ${ }^{2 *}$

\begin{abstract}
Background: Glutathione S transferase (GST) polymorphisms have been considered as risk factors for age-related cataracts, but the results remain controversial. In this study, we have performed a meta-analysis to evaluate the association between polymorphisms of GSTM1 and GSTT1 and cataract risk.

Methods: Published literature from PubMed and other databases were retrieved. The case-control studies regarding the association between GSTM1 or GSTT1 polymorphism and cataract risk were included. Pooled odds ratio (OR) and $95 \%$ confidence interval (Cl) were calculated using random- or fixed-effects model.

Results: Fifteen studies on GSTM1 (3,065 patients and 2,105 controls), and nine studies on GSTT1 (2,374 patients and 1,544 controls) were included. By pooling all the studies, GSTM1 null polymorphism was not associated with cataract risk, and this negative association maintained in subgroup analyses. However, GSTT1 null polymorphism was significantly associated with increased risk of posterior subcapsular (OR, 1.42; $95 \% \mathrm{Cl}$, 1.04-1.94) but not other subtypes of cataract. Stratified analyses demonstrated an association of GSTT1 null genotype with increased risk of cataract in Asian (OR, 1.44; 95 \% Cl, 1.14-1.83) but not Caucasian populations. In addition, seven pooled studies showed no association of cataract risk with the combined GSTM1 and GSTT1 null genotypes.

Conclusions: This meta-analysis suggests that GSTT1 null polymorphism is associated with increased risk of posterior subcapsular cataract. Given the limited sample size, the association between GSTT1 null polymorphism and cataract risk in Asian awaits further investigation.
\end{abstract}

Keywords: Glutathione S Transferases, Polymorphisms, Cataract, Meta-analysis

\section{Background}

Cataract is the opacification of eye lens with the breakdown of the lens protein microarchitecture, which adversely affects the transmission of light onto the retina [1]. Recent data suggest that cataract remains the leading cause of blindness worldwide, and the age-related cataract accounts for approximately $50 \%$ of blindness cases [2]. Epidemiologic studies have revealed some environmental risk factors for age-related cataract, including ultraviolet B light exposure, ionizing radiation, smoking, and use of steroids [3]. Recently, genetic factors have

\footnotetext{
*Correspondence: DrYeShen@163.com; chenguangdi@gmail.com ${ }^{\dagger}$ Equal contributors

'Department of Ophthalmology, The First Affiliated Hospital, Zhejiang University School of Medicine, 79 Qingchun Road, Hangzhou 310003, China ${ }^{2}$ Department of Public Health, Zhejiang University School of Medicine, 866 Yuhangtang Road, 310058 Hangzhou, China
}

been found to play important roles in the pathogenesis of age-related cataract [4]; furthermore, gene polymorphisms have been reported to be associated with agerelated cataract risk $[5,6]$.

It has been reported that oxidative stress contributes to development of age-related cataract [7]. Biochemical evidence demonstrates that generation of excessive reactive oxygen species (ROS) results in abnormal degradation, cross linking, and aggregation of lens proteins, and is involved in cataractogenesis [8]. The oxidative damage during cataractogenesis can be alleviated by cellular defense mechanisms, including catalase, superoxide dismutase, glutathione peroxidase, and glutathione $\mathrm{S}$ transferases (GSTs) in the eye [9]. Among them, GSTs are a superfamily of enzymes that play important roles in the detoxification, elimination of xenobiotics and 
antioxidation, such as carcinogens, toxins, oxidants and drugs [10]. This enzymatic superfamily is composed of three different families: mitochondrial, microsomal and cytosolic. The cytosolic family of GSTs are classified in seven classes based on chromosomal location and on sequence similarity: alpha (GSTA), mu (GSTM), pi (GSTP), theta (GSTT), kappa (GSTK), zeta (GSTZ) and omega (GSTO) [11].

Previous studies have identified numerous variants in GST genes, and some of these polymorphisms are functional, e.g., GSTT1 and GSTM1 null polymorphisms [12]. In fact, the deletion of GSTT1 or GSTM1 results in dysfunction of their enzyme activity [12], and these polymorphisms of GST are associated with increased risks of various pathologies including cancers [13] and ophthalmologic problems such as glaucoma [14]. The relationships between GST polymorphisms and risks of age-related cataract have been studied for many years, and an early meta-analysis suggested that GSTM1 and
GSTT1 null genotypes were associated with increased risk for senile cataract in Asians but not Caucasians [6]. However, recent studies showed that GSTM1 positive $\left(\mathrm{GSTM1}^{+/+}\right)$genotype was associated with a susceptibility to age-related cortical cataract in Asians [15], while GSTM1 or GSTT1 null genotype was associated with age-related cataract risk in Caucasians $[16,17]$. These inconsistent results may be due to the relatively small size of study populations from each individual study, or limited studies included by the previous meta-analysis; therefore, in this study we have conducted an update meta-analysis to reevaluate the associations between GSTM1 and GSTT1 polymorphisms and age-related cataract risk.

\section{Methods}

Identification of eligible studies

To identify all articles that evaluated the association of GST polymorphism with cataract, we carried out a literature search in the PubMed databases up to December

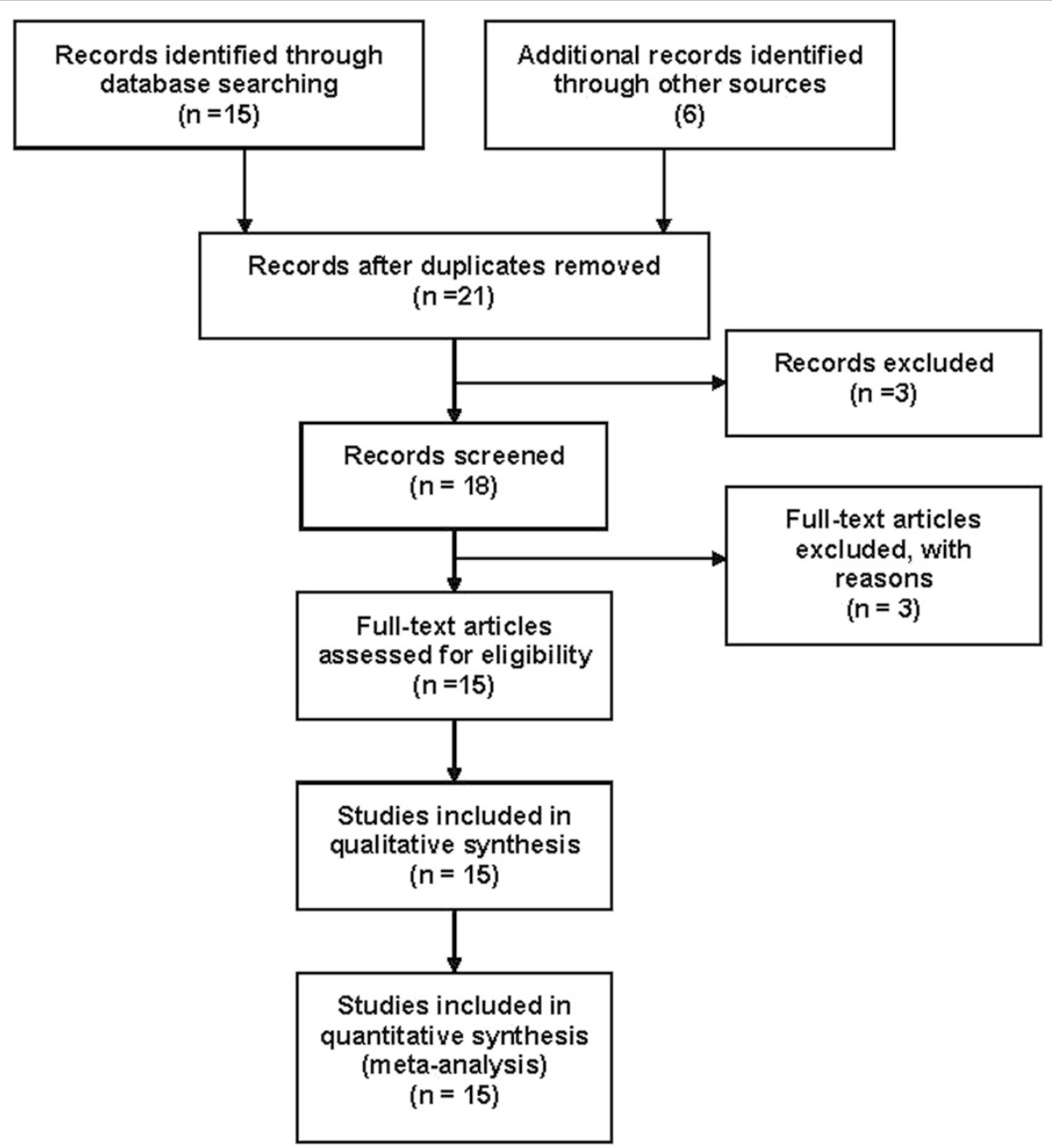

Fig. 1 Flow diagram of studies identification 
2014 with the following MeSH terms and keywords: "cataract", "glutathione S transferase", and "polymorphism". The manual search was conducted to identify additional studies from other sources (e.g., Embase, Web of Knowledge, China National Knowledge Infrastructure), review articles on this topic or references to original studies. The inclusion criteria for eligible studies included in this metaanalysis as follows: (a) a study evaluating the association between GSTM1 or GSTT1 null polymorphism and cataract, (b) a case-control study, (c) an unrelated study, if studies had partly overlapped subjects, only the one with a larger sample size was selected, (d) a study with available genotype frequency, and (e) a study with sufficient data for estimating odds ratio (OR) and $95 \%$ confidence interval (CI). Our meta-analysis was in accordance with PRISMA guidelines

Because the data included in this study were retrieved from the literatures, written informed consent for participation and ethical approval have been provided by original studies. Thus, all investigations analyzed in this meta-analysis have been carried out in compliance with the Helsinki Declaration.

\section{Data extraction}

Two investigators (W.S. and L.S) independently assessed the articles for inclusion, and reached a consensus on data extracted. For each study, the following information was extracted: the first author name and publication year of the article; ethnicity (country) of study subjects; gene polymorphisms and genotype frequencies; sample size (numbers of cases and controls); sources of controls; subtypes of cataract classified. The missing data and information of included studies were obtained by contacting the study authors through email.

\section{Statistical analysis}

The association between GSTM1, or GSTT1 polymorphism and cataract was estimated by calculating pooled OR

Table 1 Characteristics of literatures included in the meta-analysis

\begin{tabular}{|c|c|c|c|c|c|}
\hline Author/ Year & Country & Ethnicity & Sample size Cases/controls ${ }^{a}$ & Source of controls & Cataract subtype \\
\hline \multicolumn{6}{|l|}{ GSTM1 } \\
\hline Sekine 1995 [36] & Japan & Asian & 138/62 (101/30) & PB & Not classified \\
\hline Alberti 1996 [35] & United States & Caucasian & 202/98 (99/49) & $\mathrm{HB}$ & $\mathrm{NC} / \mathrm{CC} / \mathrm{M}$ \\
\hline Pi 1996 [34] & China & Asian & $59 / 112(41 / 57)$ & $H B$ & Not classified \\
\hline Hao 1999 [33] & China & Asian & $77 / 76(41 / 35)$ & $\mathrm{HB}$ & Not classified \\
\hline Juronen 2000 [25] & Estonia & Caucasian & $503 / 202(240 / 111)$ & $H B$ & $\mathrm{CC} / \mathrm{NC} / \mathrm{PSC} / \mathrm{M}$ \\
\hline Saadat 2004 [24] & Iran & Caucasian & 150/150 (90/58) & $H B$ & Not classified \\
\hline Saadat 2006 [23] & Iran & Caucasian & $95 / 95(56 / 36)$ & $H B$ & Not classified \\
\hline Guven 2007 [18] & Turkey & Caucasian & 195/136 (105/58) & $\mathrm{HB}$ & $\mathrm{CC} / \mathrm{NC} / \mathrm{PSC} / \mathrm{MC}$ \\
\hline Xu 2007 [32] & China & Asian & $120 / 118(81 / 60)$ & $H B$ & Not classified \\
\hline Azeem 2009 [22] & Egypt & Caucasian & $53 / 73(23 / 46)$ & $H B$ & Not classified \\
\hline Zhou 2010 [21] & China & Asian & 279/145 (171/95) & PB & Not classified \\
\hline Sireesha 2012 [16] & India & Caucasian & 455/205 (177/94) & PB & $\mathrm{CC} / \mathrm{NC} / \mathrm{PSC} / \mathrm{MC}$ \\
\hline Saadat 2012 [17] & Iran & Caucasian & 186/195 (104/89) & $H B$ & Not classified \\
\hline Jiang 2012 [15] & China & Asian & $422 / 312(176 / 173)$ & $H B$ & CC \\
\hline Chandra 2014 [31] & India & Caucasian & $124 / 126(43 / 68)$ & $\mathrm{HB}$ & Not classified \\
\hline \multicolumn{6}{|l|}{ GSTT1 } \\
\hline Juronen $2000^{\text {[ 25] }}$ & Estonia & Caucasian & $503 / 202(73 / 36)$ & $\mathrm{HB}$ & $\mathrm{CC} / \mathrm{NC} / \mathrm{PSC} / \mathrm{MC}$ \\
\hline Saadat 2004 [24] & Iran & Caucasian & $150 / 150(49 / 46)$ & $\mathrm{HB}$ & Not classified \\
\hline Guven 2007 [18] & Turkey & Caucasian & $195 / 136(29 / 22)$ & $H B$ & CC/NC/PSC/MC \\
\hline Azeem 2009 [22] & Egypt & Caucasian & $53 / 73(16 / 21)$ & $H B$ & Not classified \\
\hline Zhou 2010 [21] & China & Asian & $279 / 145(146 / 60)$ & PB & CC/NC/PSC \\
\hline Sireesha 2012 [16] & India & Caucasian & 455/205 (123/40) & $\mathrm{PB}$ & CC/NC/PSC/MC \\
\hline Saadat 2012 [17] & Iran & Caucasian & 186/195 (49/57) & $H B$ & Not classified \\
\hline Jiang 2012 [15] & China & Asian & $422 / 312(221 / 138)$ & $H B$ & CC \\
\hline Chandra $2014^{\text {[31] }}$ & India & Caucasian & $131 / 126(18 / 5)$ & $\mathrm{HB}$ & Not classified \\
\hline
\end{tabular}

Abbreviations: PB population-based, HB hospital-based, CC cortical cataract, NC nuclear cataract, PSC posterior sub-capsular cataract, $M C$ mixed cataract ${ }^{a}$ The number of null genotype cases or controls was presented in parenthesis 
Table 2 Association between GSTM1 or GSTT1 polymorphism and cataract risk

\begin{tabular}{|c|c|c|c|c|}
\hline Groups & $\mathrm{N}^{\mathrm{a}}$ & Statistical method ${ }^{b}$ & OR $(95 \% \mathrm{Cl})$ & $P$ \\
\hline \multicolumn{5}{|l|}{$\overline{\text { GSTM1 }}$} \\
\hline All & 15 & Random $(P<0.001)$ & $1.17(0.88-1.57)$ & 0.288 \\
\hline \multicolumn{5}{|l|}{ Ethnics } \\
\hline Caucasian & 9 & Random $(P<0.001)$ & $1.07(0.753-1.53)$ & 0.712 \\
\hline Asian & 6 & Random $(P<0.001)$ & $1.37(0.79-2.40)$ & 0.266 \\
\hline \multicolumn{5}{|l|}{ Study design } \\
\hline Population-based & 3 & Random $(P=0.001)$ & $1.17(0.58-2.33)$ & 0.666 \\
\hline Hospital-based & 12 & Random $(P<0.001)$ & $1.18(0.84-1.65)$ & 0.350 \\
\hline \multicolumn{5}{|l|}{ Gender } \\
\hline Male & 5 & Random $(P=0.035)$ & $0.89(0.58-1.37)$ & 0.598 \\
\hline Female & 5 & Random $(P<0.001)$ & $1.02(0.44-2.32)$ & 0.970 \\
\hline \multicolumn{5}{|l|}{ Subtype } \\
\hline Cortical & 4 & Random $(P=0.086)$ & $0.85(0.59-1.23)$ & 0.386 \\
\hline Nuclear & 4 & Random $(P=0.084)$ & $0.97(0.62-1.52)$ & 0.904 \\
\hline Posterior subcapsular & 3 & Fixed $(P=0.242)$ & $0.98(0.72-1.32)$ & 0.879 \\
\hline Mixed & 4 & Random $(P=0.040)$ & $0.94(0.60-1.48)$ & 0.792 \\
\hline \multicolumn{5}{|l|}{ GSTT1 } \\
\hline All & 9 & Random $(P=0.049)$ & $1.20(0.96-1.51)$ & 0.105 \\
\hline \multicolumn{5}{|l|}{ Ethnics } \\
\hline Caucasian & 7 & Random $(P=0.058)$ & $1.11(0.83-1.49)$ & 0.474 \\
\hline Asian & 2 & Fixed $(P=0.653)$ & $1.44(1.14-1.83)$ & 0.003 \\
\hline \multicolumn{5}{|l|}{ Study design } \\
\hline Population-based & 2 & Fixed $(P=0.952)$ & $1.54(1.16-2.05)$ & 0.003 \\
\hline Hospital-based & 7 & Random $(P=0.063)$ & $1.10(0.84-1.45)$ & 0.498 \\
\hline \multicolumn{5}{|l|}{ Gender } \\
\hline Male & 5 & Fixed $(P=0.984)$ & $1.29(0.98-1.70)$ & 0.073 \\
\hline Female & 5 & Fixed $(P=0.359)$ & $1.28(0.97-1.69)$ & 0.078 \\
\hline \multicolumn{5}{|l|}{ Subtype } \\
\hline Cortical & 4 & Fixed $(P=0.186)$ & $1.09(0.82-1.45)$ & 0.555 \\
\hline Nuclear & 4 & Random $(P=0.062)$ & $0.92(0.52-1.62)$ & 0.774 \\
\hline Posterior subcapsular & 4 & Fixed $(P=0.219)$ & $1.42(1.04-1.94)$ & 0.026 \\
\hline Mixed & 3 & Random $(P=0.097)$ & $1.21(0.66-2.20)$ & 0.535 \\
\hline
\end{tabular}

${ }^{a} \mathrm{~N}$ : The number of included studies

${ }^{\mathrm{b}} \mathrm{A}$ random-effects or fixed-effects model was used in presence $(P \leq 0.10)$ or absence $(P>0.10)$ of heterogeneity of included studies and the $P$ value was presented in parenthesis

and $95 \% \mathrm{CI}$. The significance of the pooled OR was determined by $\mathrm{Z}$ test, in which the $P<0.05$ was considered statistically significant. The risk of GSTM1 or GSTT1 null genotype on cataract was evaluated by comparing to wild type homozygote as their reference. Stratified analyses were also performed by ethnicity of study populations, the source of controls, gender of subjects, and cataract subtype. Considering the possible additive effect of different GST genotypes, we next evaluated the association between the genotype profile and cataract risk, in which the individuals with two putative low-risk genotypes, i.e.,, the presence of functional GSTM1 and GSTT1 alleles, were used as reference group [18]. For the quantitative synthesis analysis, the environmental effects were not adjusted due to the lack of information from the original study. The $\mathrm{I}^{2}$-based $\mathrm{Q}$ statistic test was applied to examine variations due to heterogeneity rather than chance. A random-effects (DerSimonian-Laird method) model or fixed-effects (Mantel-Haenszel method) model was applied to calculate pooled effect estimates in the presence $(P \leq 0.10)$ or absence $(P>0.10)$ of heterogeneity. The Egger's test [19] and the Begg's [20] test were applied to detect publication bias for the overall pooled analysis of GSTM1 or GSTT1 null genotypes. Additionally, the Begg's funnel plot was obtained, in which an asymmetry of the funnel plot indicates a potential publication bias. The one-way sensitivity analysis was performed when one single study was excluded each time, and the new pooled results reflect the influence of the study deleted to the overall OR. All analyses were carried out with Stata software (version 11.0; Stata Corp LP, College Station, TX), and the two-sided $P$ values were applied.

\section{Results}

\section{Characteristics of studies}

By searching PubMed, fifteen abstracts were retrieved through the search "cataract" "glutathione S transferase" and "polymorphism", and nine studies meeting the inclusion criteria were identified as eligible [15-18, 21-25]. Out of the fifteen, one was meta-analysis [6] and one was laboratory study [26]. One article was excluded due to investigation on an association of presenile cataracts with heterozygosity for galactosaemic states and with riboflavin deficiency [27]. We excluded two articles on the relationship between GST polymorphisms and risk of age-related macular degeneration [28] or primary open-angle glaucoma [29]. We also excluded one article that examined the association of GSTO polymorphisms with cataract risk [30]. In addition, we included six eligible articles with manual searching [31-36]. As a result, a total of fifteen articles on GSTM1 or GSTT1 polymorphisms meeting the inclusion criteria were identified as eligible studies (Fig. 1).

Fifteen studies on GSTM1 (3,065 cases and 2,105 controls), and nine studies on GSTT1 (2,374 cases and 1,544 controls) were included in this meta-analysis. For the ethnicities, six studies of Asians and eight studies of Caucasians were included on the GSTM1 genotype. As to GSTT1, two studies of Asians and six studies of Caucasians were included. We also grouped studies with different 
sources of controls (i.e., population-based or hospitalbased), gender (male or female) and subtypes of cataracts (e.g., cortical, nuclear, posterior sub-capsular or mixed cataract). In addition to the study by Juronen et al. [25] that determined the GSTM1 and GSTT1 phenotypes by enzyme-linked immunosorbent assay (ELISA), the genotyping for GSTM1, or GSTT1 was determined by polymerase chain reaction (PCR) assay in all other studies. The Table 1 presents the detailed characteristics of each study included in the meta-analysis.

\section{Quantitative synthesis}

Table 2 shows the results of the meta-analysis on the association of GSTM1 or GSTT1 null polymorphism with cataract risk. When pooling all the studies, we found that GSTM1 null polymorphism was not associated with cataract risk (Fig. 2a), and this negative association maintained in either Caucasian or Asian populations (Table 2). When stratified by the source of controls, gender, or cataract subtype, no association was found between GSTM1 null polymorphism and cataract risk.

For GSTT1, the overall result showed that GSTT1 null polymorphism was significantly associated with increased risk of cataract in Asian (OR, 1.44; 95 \% CI, 1.14-1.83) but not Caucasian populations (Table 2). The positive association of GSTT1 null polymorphism with increased risk of cataract was found when pooling studies with population-based (OR, 1.54; $95 \%$ CI, 1.16-2.05) but not hospital-based controls. However, there was no association between GSTT1 null polymorphism and cataract risk in male or female subjects. Interestingly, GSTT1 null polymorphism was associated with risk of posterior subcapsular (OR, 1.42; 95 \% CI, 1.04-1.94) but not other subtypes of cataract.
We next investigated the effects of the profiles of GST genotypes on the risk of cataract, and examined the association between combinations of GSTM1 and GSTT1 null genotypes and cataract risk. Table 3 displays cataract risk associated with combinations of GST null genotypes, and the trend in risk associated with each putative high-risk null genotype. The results showed no association between the combined GSTM1 and GSTT1 null genotypes and cataract risk in all population, Caucasian or Asian population. When stratified by source of controls, pooled two studies with population-based controls showed that combination of GSTM1 null and GSTT1 positive $\left(\mathrm{GSTT1}^{+/+}\right)$genotypes played a protective role in cataract risk (OR, 0.71; $95 \%$ CI, 0.54-0.92), but combination of GSTM1 positive and GSTT1 null, or GSTM1 and GSTT1 null genotypes was not associated with cataract risk. The other sub-group analyses showed no association between combination of GSTM1 and GSTT1 polymorphisms and cataract risk.

\section{Potential publication bias and sensitivity analysis}

We firstly detected the publication bias by the Begg's test for the overall pooled analyses of GSTM1 and GSTT1 null genotype, and found symmetric distribution of corresponding funnel plots for GSTM1 genotype with a $P$ value of 0.138 , and GSTT1 genotype with a $P$ value of 0.754 (Fig. 3). However, the Egger's test showed that the $P$ values for GSTM1 and GSTT1 null genotype were 0.037 and 0.908 respectively, suggesting a publication bias for studies on GSTM1 but not GSTT1 genotype.

Sensitivity analysis showed that exclusion of each study did not influence the result in specific genotype comparison for GSTM1 and GSTT1 polymorphism (Fig. 4), suggesting that the results of synthetic analysis were robust.

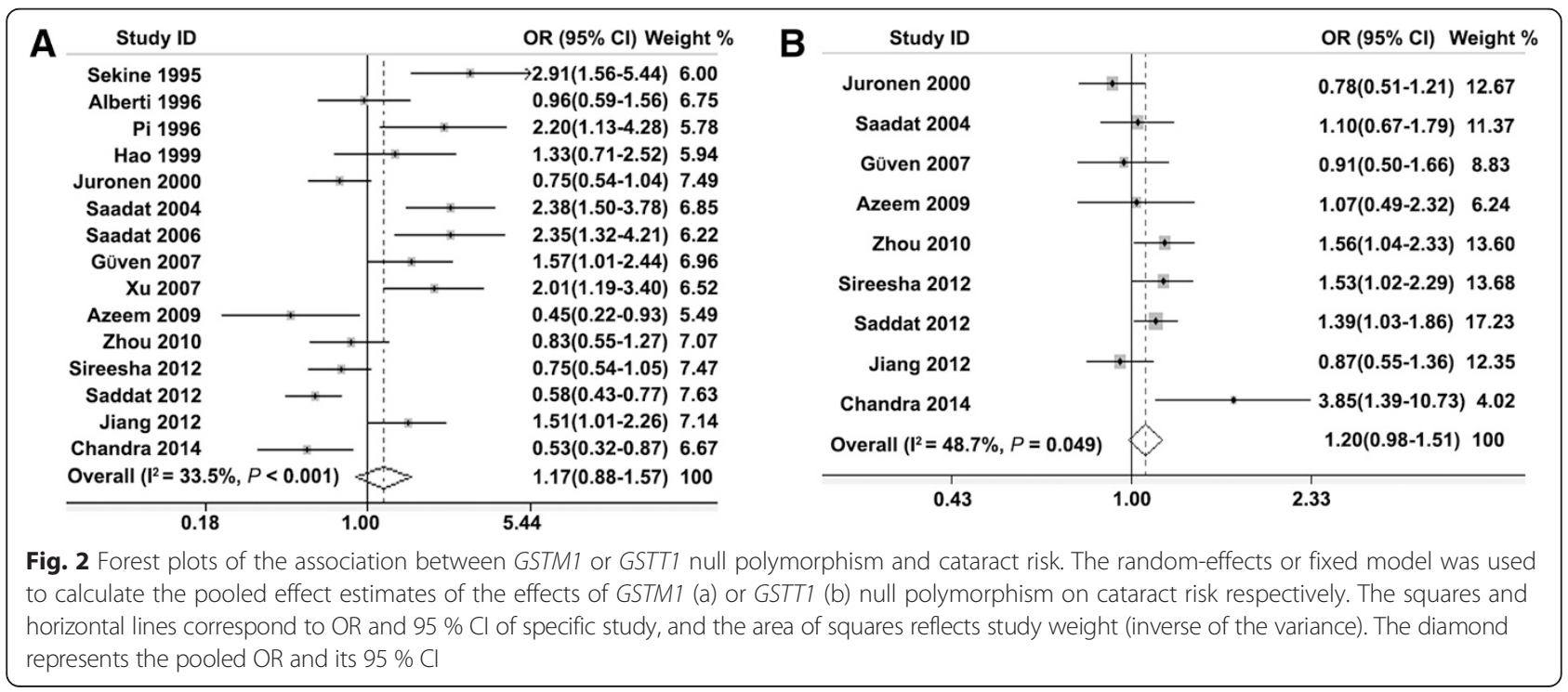


Table 3 Association between GSTM1 and GSTT1 polymorphisms and cataract risk

\begin{tabular}{|c|c|c|c|c|}
\hline Groups & Number $^{a}$ & Statistical method ${ }^{b}$ & OR $(95 \% \mathrm{Cl})$ & $P$ \\
\hline \multicolumn{5}{|l|}{ All } \\
\hline GSTM1 null + GSTT1 positive & 7 & Random $(P<0.001)$ & $0.83(0.56-1.23)$ & 0.356 \\
\hline GSTM1 positive + GSTT1 null & 7 & Fixed $(P=0.240)$ & $1.20(0.95-1.53)$ & 0.134 \\
\hline GSTM1 null + GSTT1 null & 7 & Random $(P=0.010)$ & $1.16(0.71-1.89)$ & 0.545 \\
\hline \multicolumn{5}{|l|}{ Ethnics } \\
\hline \multicolumn{5}{|l|}{ Caucasian } \\
\hline GSTM1 null + GSTT1 positive & 6 & Random $(P<0.001)$ & $0.85(0.52-1.37)$ & 0.494 \\
\hline GSTM1 positive + GSTT1 null & 6 & Fixed $(P=0.658)$ & $1.00(0.74-1.34)$ & 0.983 \\
\hline GSTM1 null + GSTT1 null & 6 & Random $(P=0.008)$ & $1.27(0.67-2.38)$ & 0.466 \\
\hline \multicolumn{5}{|l|}{ Study design } \\
\hline \multicolumn{5}{|l|}{ PB } \\
\hline GSTM1 null + GSTT1 positive & 2 & Fixed $(P=0.591)$ & $0.71(0.54-0.92)$ & 0.009 \\
\hline GSTM1 positive + GSTT1 null & 2 & Fixed $(P=0.334)$ & $1.03(0.69-1.53)$ & 0.899 \\
\hline GSTM1 null + GSTT1 null & 2 & Random $(P=0.036)$ & $0.87(0.34-2.18)$ & 0.760 \\
\hline \multicolumn{5}{|l|}{$H B$} \\
\hline GSTM1 null + GSTT1 positive & 5 & Random $(P<0.001)$ & $0.88(0.47-1.65)$ & 0.697 \\
\hline GSTM1 positive + GSTT1 null & 5 & Fixed $(P=0.196)$ & $1.32(0.97-1.79)$ & 0.073 \\
\hline GSTM1 null + GSTT1 null & 5 & Random $(P=0.024)$ & $1.38(0.71-2.69)$ & 0.336 \\
\hline \multicolumn{5}{|l|}{ Gender } \\
\hline \multicolumn{5}{|l|}{ Male } \\
\hline GSTM1 null + GSTT1 positive & 2 & Fixed $(P=0.990)$ & $0.88(0.49-1.59)$ & 0.676 \\
\hline GSTM1 positive + GSTT1 null & 2 & Fixed $(P=0.476)$ & $0.84(0.28-2.50)$ & 0.749 \\
\hline GSTM1 null + GSTT1 null & 2 & Fixed $(P=0.672)$ & $1.48(0.52-4.21)$ & 0.463 \\
\hline \multicolumn{5}{|l|}{ Female } \\
\hline GSTM1 null + GSTT1 positive & 2 & Random $(P<0.001)$ & $0.79(0.06-10.87)$ & 0.858 \\
\hline GSTM1 positive + GSTT1 null & 2 & Fixed $(P=0.767)$ & $0.62(0.27-1.43)$ & 0.264 \\
\hline GSTM1 null + GSTT1 null & 2 & Random $(P=0.074)$ & $0.91(0.15-5.57)$ & 0.919 \\
\hline \multicolumn{5}{|l|}{ Cataract type } \\
\hline \multicolumn{5}{|l|}{ Cortical } \\
\hline GSTM1 null + GSTT1 positive & 3 & Fixed $(P=0.745)$ & $0.82(0.62-1.10)$ & 0.181 \\
\hline GSTM1 positive + GSTT1 null & 3 & Fixed $(P=0.131)$ & $1.39(0.99-1.96)$ & 0.061 \\
\hline GSTM1 null + GSTT1 null & 3 & Fixed $(P=0.171)$ & $1.03(0.72-1.48)$ & 0.855 \\
\hline \multicolumn{5}{|l|}{ Nuclear } \\
\hline GSTM1 null + GSTT1 positive & 2 & Random $(P=0.030)$ & $1.00(0.39-2.56)$ & 0.994 \\
\hline GSTM1 positive + GSTT1 null & 2 & Random $(P=0.081)$ & $0.67(0.11-4.24)$ & 0.668 \\
\hline GSTM1 null + GSTT1 null & 2 & Fixed $(P=0.868)$ & $1.16(0.56-2.38)$ & 0.694 \\
\hline \multicolumn{5}{|l|}{ Posterior subcapsular } \\
\hline GSTM1 null + GSTT1 positive & 2 & Random $(P=0.038)$ & $1.20(0.42-3.39)$ & 0.734 \\
\hline GSTM1 positive + GSTT1 null & 2 & Fixed $(P=0.157)$ & $1.15(0.59-2.26)$ & 0.682 \\
\hline GSTM1 null + GSTT1 null & 2 & Fixed $(P=0.399)$ & $1.97(0.98-3.97)$ & 0.059 \\
\hline \multicolumn{5}{|l|}{ Mixed } \\
\hline GSTM1 null + GSTT1 positive & 2 & Random $(P=0.019)$ & $0.81(0.25-2.61)$ & 0.724 \\
\hline
\end{tabular}


Table 3 Association between GSTM1 and GSTT1 polymorphisms and cataract risk (Continued)

\begin{tabular}{lllll}
\hline GSTM1 positive + GSTT1 null & 2 & Fixed $(P=0.130)$ & $1.22(0.68-2.21)$ & 0.505 \\
GSTM1 null + GSTT1 null & 2 & Fixed $(P=0.523)$ & $1.44(0.74-2.79)$ & 0.279 \\
\hline
\end{tabular}

${ }^{\mathrm{a}} \mathrm{N}$ : The number of included studies

${ }^{\mathrm{b}} \mathrm{A}$ random-effects or fixed-effects model was used in presence $(P \leq 0.10)$ or absence $(P>0.10)$ of heterogeneity of included studies and the $P$ value was presented in parenthesis

\section{Discussion}

Before inclusion of studies, we briefly searched PubMed, Embase, Web of Science and China National Knowledge Infrastructure, and found that most of studies examined association of GSTM1 or GSTT1 polymorphisms with cataract risk while very limited studies were related to other GST polymorphisms, e.g., GSTM3, GSTO or GSTP polymorphisms. Thus, this meta-analysis only evaluated the effects of GSTM1 and GSTT1 ploymorphisms on cataract risk. Our data showed that GSTT1 but not GSTM1 null polymorphism was associated with cataract risk in Asians. Although different subtypes of cataract have their own pathogenesis and clinical characteristics, our meta-analysis data indicate that GSTT1 null polymorphism may contribute to increased risk of posterior subcapsular cataract.

In 1995, Sekine and colleagues for the first time reported possible correlation of GSTM1 null genotype frequency with cataract risk [36]. However, the following studies showed inconsistent results [18, 21-25, 32-35]. By pooling these early studies, previous meta-analysis by Sun et al., did not find an association of GSTM1 null genotype with cataract risk [6]. Even including three more studies, we did not find positive relationship between GSTM1 null genotype and cataract risk. To be noted, although previous meta-analysis indicated an association of GSTM1 null genotype and increased risk of cataract in Asians [6], our data did not confirm this association when including one more study on Asians.

For GSTT1 polymorphism, pooled four early studies on Caucasian showed no association $[18,22,24,25]$ while one study on Asians [21] showed positive association between GSTT1 null genotype and cataract risk; however, by pooling these five studies, no association was found [6]. By including four recent studies, our meta-analysis showed positive association of GSTT1 polymorphism with increased risk of cataract in all populations, and this association remained in Asians when two studies were pooling $[15,21]$. Previous studies reported gender-dependent effects of GSTT1 null polymorphism on cataract risk [18, 22, 24]; however, recent two studies showed negative results $[15,16]$. We performed a subgroup analysis stratified by gender with all five studies, and results showed no significant association, which was consistent with previous meta-analysis data based on three studies [6]. In addition, our data showed positive association of GSTT1 null polymorphism with increased risk of posterior subcapsular cataract although previous pooled study indicated that this association did not reach significant (OR, 1.21; $95 \% \mathrm{CI}$, 0.96-1.53) [6]. Since the studies included for subgroup analyses were still limited, future studies are required to validate the association between GSTT1 null polymorphism and cataract risk.

To the best of our knowledge, the association between combination of GST polymorphisms and susceptibility to cataract has been assessed for the first time by our meta-analysis. The study by Juronen et al., firstly reported that the GSTM1 positive phenotype frequency was significantly higher in the cataract group than in the controls, and the cataract risk associated with the GSTM1 positive phenotype was increased in
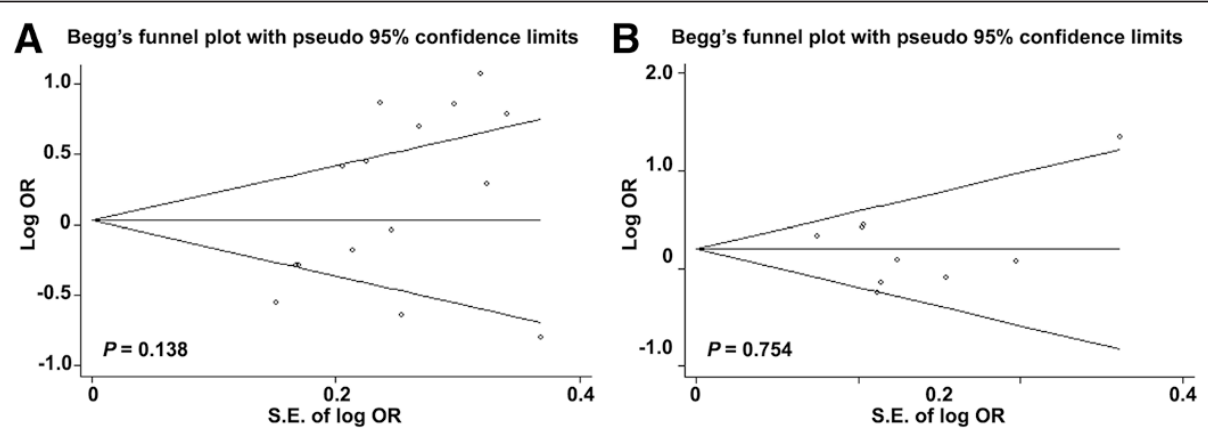

Fig. 3 Funnel plots showed symmetric distribution. Log OR is plotted against the standard error of log OR for studies on GSTM1 (a) or GSTT1 null (b) polymorphism. The dots represent specific studies for the indicated association 


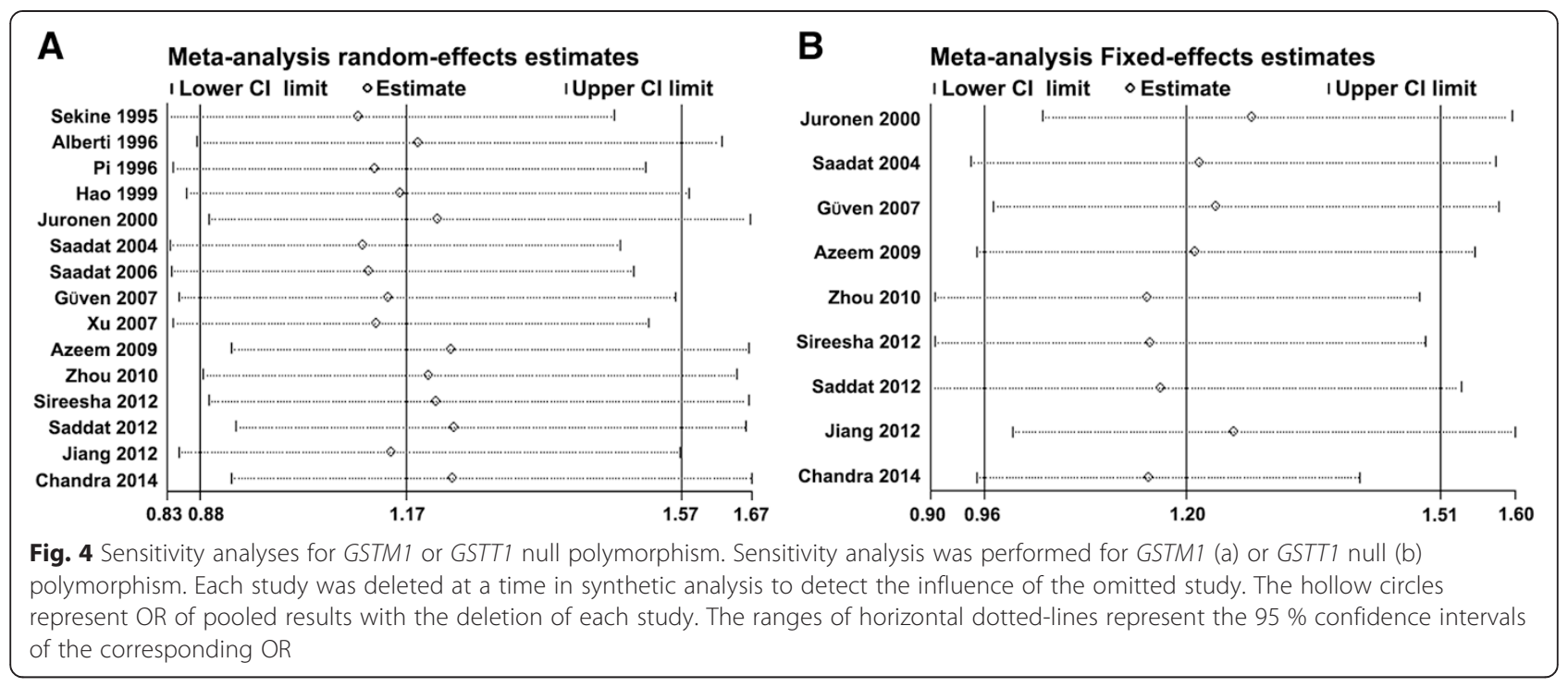

carriers of the combined GSTM1 positive and GSTT1 positive phenotypes [25]. However, a later study by Saadat et al., showed that individuals with the null genotypes for GSTM1 and GSTT1, or combination of GSTT1 positive and GSTM1 null genotypes were at a significantly higher risk for developing cataract than individuals with both the genes positive genotypes [24]. The following studies consecutively presented inconsistent results $[15,16,18,22]$. By pooling seven studies, our meta-analysis results did not show a significant association between each combination of GSTM1 and GSTT1 genotypes and cataract risk. Two pooled studies with population-based controls showed that combination of GSTM1 null and GSTT1 positive genotypes played a protective role in cataract risk [16, 25]; however, this positive association was not found in other stratified analyses. Thus, the result should be interpreted with caution.

When compared to individual studies, the metaanalysis has a vital advantages. However, some potential limitations in our study should be considered. First, the inclusion of studies might not be sufficient since we only included published papers with language in English, or Chinese. It is possible that some papers published in other languages may not indexed by the database (e.g. PubMed, Embase, Web of Science). Thus, the publication bias for GSTM1 polymorphism detected in our study might be due to insufficient inclusion of published studies. Second, this meta-analysis was limited by the small sample size, especially in subgroup analyses aforementioned (e.g., studies on GSTT1 polymorphism in Asians), and this need further investigation. Third, basic methodological differences among the studies, e.g., ELISA vs. PCR assay for genotyping, might have affected the results. Fourth, most of the studies included did not categorize the cataract patients as cortical, nuclear, posterior subcapsular and mixed cataract. Although we found positive association between GSTT1 null polymorphism and increased risk of posterior subcapsular cataract, however, only four studies with available data were pooled [16, 18, 21, 25], and thus this association awaits further confirmation. Fifth, the primary outcome measure was calculated based on individual unadjusted ORs, which might affect the evaluation precision of the study. The lack of detailed data in each study prevented multiple testing for combined effects of gene-environment factors on cataract risk, and thus future studies should address this point. Last, the Caucasian and Asian subjects from different countries might have been genetically heterogeneous, e.g., different lifestyle and environment (e.g., European vs. Arabian). These factors may explain the heterogeneity in this meta-analysis for Caucasian subjects.

\section{Conclusion}

In summary, the present meta-analysis showed that the association between GSTM1 null polymorphism and cataract risk was either negative or evidence limited. The GSTT1 null polymorphism was significantly associated with increased risk of posterior subcapsular cataract. Given the limited study populations, more studies with large study population are suggested to further validate the relationship between GST polymorphisms and genetic predisposition to cataract, e.g., association of GSTT1 null polymorphism with cataract risk in Asian. 


\section{Competing interests}

The authors declare that they have no competing interests.

\section{Authors' contributions}

Conceived and designed the study: WS, GC. Acquisition of data: WS, LS. Analysis and interpretation of data: YS, YC. Drafting the manuscript: WS, GC. Revising the manuscript critically for important intellectual content: WS, LS, YS, YC, GC. All authors read and approved the final manuscript.

\section{Acknowledgement}

This work was supported by the

National Natural Science Foundation of China (81200717), the Zhejiang Provincial Educational Bureau Foundation, China (Y201223905), and the Qianjiang Talents Program of Zhejiang Province, China (2013R10041). The study sponsors had no role in the design and conduct of the study; collection, management, analysis, and interpretation of the data; and preparation, review, or approval of the manuscript.

\section{Financial disclosure}

This work was supported by the National Natural Science Foundation of China (81200717), the Zhejiang Provincial Educational Bureau Foundation, China (Y201223905), and the Qianjiang Talents Program of Zhejiang Province, China (2013R10041). The funders have no role in study design; in the collection, analysis and interpretation of data; in the writing of the report; and in the decision to submit the article for publication.

\section{Received: 15 March 2015 Accepted: 6 July 2015}

Published online: 26 July 2015

\section{References}

1. Asbell PA, Dualan I, Mindel J, Brocks D, Ahmad M, Epstein S. Age-related cataract. Lancet. 2005;365:599-609.

2. West S. Epidemiology of cataract: accomplishments over 25 years and future directions. Ophthalmic Epidemiol. 2007;14:173-8.

3. Robman L, Taylor H. External factors in the development of cataract. Eye (Lond). 2005;19:1074-82.

4. Shiels A, Hejtmancik JF. Genetic origins of cataract. Arch Ophthalmol. 2007;125:165-73.

5. Yang J, Luo J, Zhou P, Fan Q, Luo Y, Lu Y. Association of the ephreceptor tyrosinekinase-type A2 (EPHA2) gene polymorphism rs3754334 with age-related cataract risk: a meta-analysis. PLoS One. 2013;8:e71003.

6. Sun L, Xi B, Yu L, Gao XC, Shi DJ, Yan YK, et al. Association of glutathione S-transferases polymorphisms (GSTM1 and GSTT1) with senile cataract: a meta-analysis. Invest Ophthalmol Vis Sci. 2010;51:6381-6.

7. Fletcher AE. Free radicals, antioxidants and eye diseases: evidence from epidemiological studies on cataract and age-related macular degeneration. Ophthalmic Res. 2010;44:191-8.

8. Berthoud VM, Beyer EC. Oxidative stress, lens gap junctions, and cataracts. Antioxid Redox Signal. 2009;11:339-53.

9. Vinson JA. Oxidative stress in cataracts. Pathophysiology. 2006;13:151-62.

10. Strange RC, Jones PW, Fryer AA. Glutathione S-transferase: genetics and role in toxicology. Toxicol Lett. 2000;112-113:357-63.

11. Polimanti R, Carboni C, Baesso I, Piacentini S, lorio A, De Stefano GF, et al. Genetic variability of glutathione S-transferase enzymes in human populations: functional inter-ethnic differences in detoxification systems. Gene. 2013;512:102-7.

12. Josephy PD. Genetic variations in human glutathione transferase enzymes: significance for pharmacology and toxicology. Hum Genomics Proteomics. 2010;2010:876940.

13. Di Pietro G, Magno LA, Rios-Santos F. Glutathione S-transferases: an overview in cancer research. Expert Opin Drug Metab Toxicol. 2010;6:153-70.

14. Yu Y, Weng Y, Guo J, Chen G, Yao K. Association of glutathione $S$ transferases polymorphisms with glaucoma: a meta-analysis. PLoS One. 2013;8:e54037

15. Jiang Z, Liang K, Zhang Q, Tao L. Glutathione S-transferases polymorphisms confer susceptibility to senile cortical cataract in the Han Chinese population. Mol Vis. 2012;18:1247-52.

16. Sireesha R, Laxmi SG, Mamata M, Reddy PY, Goud PU, Rao PV, et al. Total activity of glutathione-S-transferase (GST) and polymorphisms of GSTM1 and GSTT1 genes conferring risk for the development of age related cataracts. Exp Eye Res. 2012;98:67-74.
17. Saadat I, Ahmadi Z, Farvardin-Jahromi M, Saadat M. Association between cataract and genetic polymorphisms of GSTM1, GSTT1, and GSTO2 with respect of work place. Mol Vis. 2012;18:1996-2000.

18. Guven M, Unal M, Sarici A, Ozaydin A, Batar B, Devranoglu K. GlutathioneS-transferase M1 and T1 genetic polymorphisms and the risk of cataract development: a study in the Turkish population. Curr Eye Res. 2007;32:447-54.

19. Hayashino $Y$, Noguchi $Y$, Fukui T. Systematic evaluation and comparison of statistical tests for publication bias. J Epidemiol. 2005;15:235-43.

20. Begg CB, Mazumdar M. Operating characteristics of a rank correlation test for publication bias. Biometrics. 1994;50:1088-101.

21. Zhou J, Hu J, Guan H. The association between copy number variations in glutathione S-transferase M1 and T1 and age-related cataract in a Han Chinese population. Invest Ophthalmol Vis Sci. 2010;51:3924-8.

22. Abdel Azeem AA, Mahmoud AA, Salaheldine MM, Amr K. Implication of glutathione S-transferase M1 and T1 polymorphisms in the development of senile cataract among Egyptians. Bratisl Lek Listy. 2009;110:678-83.

23. Saadat M, Farvardin-Jahromi M. Occupational sunlight exposure, polymorphism of glutathione S-transferase M1, and senile cataract risk. Occup Environ Med. 2006;63:503-4.

24. Saadat M, Farvardin-Jahromi M, Saadat H. Null genotype of glutathione S-transferase M1 is associated with senile cataract susceptibility in non-smoker females. Biochem Biophys Res Commun. 2004;319:1287-91.

25. Juronen E, Tasa G, Veromann S, Parts L, Tiidla A, Pulges R, et al. Polymorphic glutathione S-transferases as genetic risk factors for senile cortical cataract in Estonians. Invest Ophthalmol Vis Sci. 2000;41:2262-7.

26. Bu L, Yan S, Jin M, Jin Y, Yu C, Xiao S, et al. The gamma S-crystallin gene is mutated in autosomal recessive cataract in mouse. Genomics. 2002;80:38-44.

27. Prchal JT, Conrad ME, Skalka HW. Association of presenile cataracts with heterozygosity for galactosaemic states and with riboflavin deficiency. Lancet. 1978;1:12-3.

28. Othman H, Gholampour AR, Saadat I, Farvardin-Jahromoi M, Saadat M. Age-related macular degeneration and genetic polymorphisms of glutathione S-transferases M1 (GSTM1) and T1 (GSTT1). Mol Biol Rep. 2012;39:3299-303.

29. Juronen E, Tasa G, Veromann S, Parts L, Tiidla A, Pulges R, et al. Polymorphic glutathione S-transferase $\mathrm{M} 1$ is a risk factor of primary open-angle glaucoma among Estonians. Exp Eye Res. 2000;71:447-52.

30. Stamenkovic M, Radic T, Stefanovic I, Coric V, Sencanic I, Pljesa-Ercegovac M, et al. Glutathione S-transferase omega-2 polymorphism Asn142Asp modifies the risk of age-related cataract in smokers and subjects exposed to ultraviolet irradiation. Clin Experiment Ophthalmol. 2013:42:277-83.

31. Chandra A, Raza ST, Abbas S, Singh L, Rizvi S, Ahmed F et al. Polymorphism of GST and FTO Genes in Risk Prediction of Cataract among a North Indian Population. Ophthalmic Genet. In press.

32. Xu M, Fu S, L Z. Relationship between GSTM1 gene deletion and susceptibility to senile cataract. Acta Acad Med Jiangxi. 2007;47:33-8.

33. Hao Y, He S, Gu Z, Zhao Y, Li X, Wang C, et al. Relationship between GSTM1 genotype and susceptibility to senile cataract. Zhonghua Yan Ke Za Zhi. 1999;35:104-6.

34. Pi J, Bai Y, Zheng Q. A study on relationship between glutathione S-transferase mu gene deletion and senile cataract susceptibility. Zhonghua Yan Ke Za Zhi. 1996:32:224-6.

35. Alberti G, Oguni M, Podgor M, Sperduto RD, Tomarev S, Grassi C, et al. Glutathione S-transferase M1 genotype and age-related cataracts. Lack of association in an Italian population. Invest Ophthalmol Vis Sci. 1996;37:1167-73.

36. Sekine $Y$, Hommura S, Harada S. Frequency of glutathione-S-transferase 1 gene deletion and its possible correlation with cataract formation. Exp Eye Res. 1995;60:159-63. 